\title{
Heart ventricular activation in VAT difference maps from children with chronic kidney disease
}

\author{
Krystyna Laszki-Szcząchor • Dorota Polak-Jonkisz • \\ Danuta Zwolińska • Lesław Rusiecki • Anna Janocha • \\ Małgorzata Sobieszczańska
}

Received: 26 February 2011 /Revised: 16 June 2011 / Accepted: 18 July 2011 / Published online: 11 August 2011

(C) The Author(s) 2011. This article is published with open access at Springerlink.com

\begin{abstract}
Children with chronic kidney disease (CKD) are affected by cardiovascular complications, including disturbances in the intraventricular conduction system. Body surface potential mapping (BSPM) is a non-invasive method of assessing the cardioelectrical field. Our aim was to investigate conduction disturbances in young CKD patients using ventricular activation time (VAT) maps. Our study comprised 22 CKD children (mean age: 13.1土 2.5 years) treated conservatively and 29 control patients. For each child 12-lead electrocardiogram (ECG) readings were taken, and blood pressure and serum concentrations of iPTH, Pi, t-Ca, creatinine, $\mathrm{Fe}^{+3}$, ferritin, and $\mathrm{Hb}$, as well as eGFR were measured. All children underwent registration in the 87-lead BSPM system, and group-mean VAT maps and a difference map, which presents statistically significant differences between the groups, were created. The VAT map distribution in CKD patients revealed abnormalities specific to left anterior fascicle block. The difference map displays the areas of intergroup VAT changes, which are of discriminative value in detecting intraventricular conduction disturbances. Intraventricular conduction impairments in the left bundle branch may occur in children
\end{abstract}

K. Laszki-Szcząchor · L. Rusiecki • M. Sobieszczańska $(\bowtie)$ Department of Pathophysiology, Wroclaw Medical University, Marcinkowskiego Street 1,

50-368 Wroclaw, Poland

e-mail: malsobie@poczta.onet.pl

D. Polak-Jonkisz $\cdot$ D. Zwolińska

Department of Pediatric Nephrology,

Wroclaw Medical University,

Wroclaw, Poland

A. Janocha

Department of Physiology, Wroclaw Medical University,

Wroclaw, Poland with CKD. BSPM enables conduction disturbances in CKD children to be detected earlier than using 12-lead ECG. The difference map derived from the group-mean isochrone maps precisely localizes the sites of disturbed conduction in the heart intraventricular conduction system.

Keywords Chronic kidney disease $\cdot$ Body surface potential mapping $\cdot$ Isochrone map $\cdot$ Ventricular activation time

\section{Introduction}

There are numerous cardiovascular complications of compound pathogenesis that appear in children with conservatively treated chronic kidney disease (CKD). The causes of these complications can be metabolic, hemodynamic, toxic, humoral or vascular. Clinical observations indicate the importance of arranging effective treatment for cardiovascular disorders and predicting a prospective course of the disease [1].

In our previous investigations concerning pediatric patients with CKD, disorders in the heart intraventricular conduction system were discovered using isointegral maps [2].

Body surface potential mapping (BSPM) is one of the electrocardiographic methods applied in modern cardiac diagnostics. It enables the detection of various heart abnormalities, as shown by many investigators, including Miyashita and Okano, and Green and Abildskov [3, 4]. Some reports have stated that BSPM is clinically useful in the early and precise diagnosis of intraventricular conduction disturbances, owing to its superiority over standard 12lead ECG [5].

The BSPM technique is based upon simultaneous registering of electrocardiographic signals from a multi- 
electrode body surface array. Electrodes cover the surface of the thorax, which enhances the possibility of detecting local events in the electrocardiographic field. The large number of recording electrode sites results in higher sensitivity of BSPM, giving it an advantage over standard ECG. The results of BSPM examinations are displayed graphically in the form of so-called "heart maps," which comprise three different types: isochrone, isopotential, and isointegral. The first map reflects the propagation of the heart depolarization over the thoracic surfaces in time, the second shows the distribution of the instant heart potential over the thorax, and the third type depicts resultant potential fluctuations occurring within the given time intervals of the cardiac cycle (area under the ECG curve).

In the present study we used isochrone maps, which reflect the body surface distribution of ventricular activation time (VAT) isolines. The isochrones are lines connecting all sites of the myocardium that are activated at the same time, and isochrone maps (or VAT maps) provide general information about the ventricular activation sequence. The distribution and values of isochrone maps precisely reflect the time of ventricular activation propagation [3-5].

In order to extract divergences between the VAT maps of the two groups examined, i.e., children with CKD and children without CKD, a "difference map" was created.

The aim of the present study was to investigate possible intraventricular conduction disturbances in young patients with CKD using VAT maps.

\section{Patients and methods}

A group of 22 children with CKD (mean age: $13.1 \pm 2.5$ years) treated conservatively, were recruited to the study and constituted Group S. These 22 children were subdivided according to CKD progression, following the K/DOQI 2002 guidelines [6]. Of the study patients, 18 were at the third stage of CKD and the remaining 4 were at the second stage. The duration of third-stage CKD was 2.3-7.1 years (mean: $4.94 \pm 1.48$ years), and the duration of second-stage CKD was $0.9-5.0$ years (mean: $2.71 \pm 1.17$ years).

In the study group, several primary causes of CKD were noted: chronic glomerulonephritis (13 patients), reflux nephropathy (7 patients), and congenital anomalies of the urinary tract: polycystic kidney disease and obstructive uropathy (2 patients).

Seven of the CKD patients were treated with antihypertensive drugs (calcium channel blockers, angiotensinconverting enzyme $[\mathrm{ACE}]$ inhibitors, beta-blocker agents). Five were treated with monotherapy (calcium channel blockers or ACE inhibitors) and the remaining two with combined therapy (calcium channel blockers with betablockers or calcium channel blockers with ACE inhibitors).
The other drugs used in the therapy were: vitamin $D_{3}$ analogues, vitamins C, B complex, and folic acid, as well as iron and ranitidine hydrochloride or omeprazole.

All the patients with third-stage CKD were kept on phosphate-poor diets (phosphate intake did not exceed 500 $800 \mathrm{mg} /$ day) and were treated with compounds that bind phosphate in the intestine (calcium carbonate in doses of $35-200 \mathrm{mg} / \mathrm{kg} /$ day). The doses of these therapeutic agents were individualized, so that the serum concentration of phosphate was between 4.5 and $5.52 \mathrm{mg} / \mathrm{dl}$ and calcium was between 8.8 and $10.8 \mathrm{mg} / \mathrm{dl}$. Dietary calcium intake was defined as: 500-600 $\mathrm{mg}$ of elementary calcium per day.

In each CKD patient, 12-lead ECG examination was carried out. No signs of conduction abnormalities were observed on the ECG tracings from any of the patients.

For all CKD patients, the trans-chest echocardiographic examination (ECHO) was made with Sonos 1000 apparatus (Hewlett Packard), equipped with 3.0-MHz topographic heads. It was found that the M-mode measurements of the left ventricle (late diastolic and late systolic diameter, interventricular wall, and left ventricle posterior wall thickness) were within the normal ranges after adjusting for the patients' body mass. Thus, there were no cases of left ventricle hypertrophy in the entire study population. Also, the markers of overall contractility, i.e., ejection fraction (EF) and shortening fraction (FS), indicated quite normal systolic function of the left ventricle in all the patients examined.

The control group (Group C) comprised 29 children (mean age: $12.7 \pm 3.1$ years) hospitalized because of nocturnal enuresis and presenting no renal or cardiac clinical symptoms, as well as showing normal arterial blood pressure values and no abnormalities on ECG and ECHO findings.

In all patients, the following parameters were determined in the serum: inorganic phosphate $\left(\mathrm{P}_{\mathrm{I}}\right)$, total calcium $(\mathrm{t}-\mathrm{Ca})$, creatinine (cr), intact parathyroid hormone (iPTH), iron $\left(\mathrm{Fe}^{+3}\right)$, ferritin, and hemoglobin $(\mathrm{Hb})$. The estimated glomerular filtration rate (eGFR) was calculated according to the Schwartz formula [7]. Serum $\mathrm{P}_{\mathrm{I}}$, and t-Ca were tested by the direct potentiometry method, using ion-selective ISE electrodes with the automatic analyzer Konelab (bioMérieux, France) with the following reference ranges: Ca-t: 8.80 $10.80 \mathrm{mg} / \mathrm{dl} ; \mathrm{P}_{\mathrm{I}}: 4.5-5.52 \mathrm{mg} / \mathrm{dl}$. Serum creatinine was determined by the Jaffe method (reference range: 0.3$1.0 \mathrm{mg} / \mathrm{dl}$ ). iPTH was estimated by the immunoenzymatic ELISA microassay of the solid phase from Roche Diagnostic (Poland) on an Immulite 2000 apparatus (reference range: 11-67 pg/ml). The colorimetric method was used for determination of serum iron (reference range 8.9$21.4 \mu \mathrm{mol} / \mathrm{l}$ ). Ferritin was tested by nefelometric methods (reference range: $7-290 \mu \mathrm{g} / \mathrm{l}$ ).

Table 1 displays the selected demographic and biochemical parameters taken from the study groups examined. 
Table 1 Biochemical findings in the groups examined

\begin{tabular}{lccc}
\hline Parameters & $\begin{array}{l}\text { C, control group } \\
n=29\end{array}$ & $\begin{array}{l}\mathrm{S}, \text { study group } \\
n=22\end{array}$ & $p$ \\
\hline Age (years) & $12.7 \pm 3.1$ & $13.1 \pm 2.5$ & $\mathrm{NS}$ \\
$\mathrm{F} / \mathrm{M}$ & $13 / 16$ & $17 / 5$ & $<0.05$ \\
$\mathrm{BMI}$ & $20.59 \pm 1.03$ & $19.05 \pm 2.82$ & $<0.02$ \\
$\mathrm{iPTH}(\mathrm{pg} / \mathrm{ml})$ & $41.53 \pm 4.33$ & $91.00 \pm 5.21$ & $<0.001$ \\
$\mathrm{P}_{\mathrm{I}}(\mathrm{mEq} / \mathrm{l})$ & $4.87 \pm 0.31$ & $5.01 \pm 0.45$ & $\mathrm{NS}$ \\
$\mathrm{t}-\mathrm{Ca}(\mathrm{mg} / \mathrm{dl})$ & $9.65 \pm 0.71$ & $10.02 \pm 0.34$ & $<0.05$ \\
$\mathrm{cr}(\mathrm{mg} / \mathrm{dl})$ & $0.78 \pm 0.22$ & $1.33 \pm 0.45$ & $<0.001$ \\
$\mathrm{Hb}(\mathrm{g} / \mathrm{dl})$ & $13.96 \pm 0.64$ & $13.71 \pm 0.74$ & $\mathrm{NS}$ \\
$\mathrm{Fe}(\mu \mathrm{mol} / \mathrm{l})$ & $16.31 \pm 3.50$ & $13.28 \pm 4.94$ & $<0.04$ \\
Ferritine $(\mu \mathrm{g} / \mathrm{l})$ & $119.11 \pm 14.08$ & $115.38 \pm 37.61$ & $\mathrm{NS}$ \\
$\mathrm{GFR}(\mathrm{ml} / \mathrm{min} / 1.73 \mathrm{~m})$ & $>90 \mathrm{ml}$ & $45.00 \pm 9.00 \mathrm{ml}$ & $<0.001$ \\
Systolic BP $(\mathrm{mmHg})$ & $121.18 \pm 4.34$ & $121.45 \pm 10.27$ & $\mathrm{NS}$ \\
Diastolic BP $(\mathrm{mmHg})$ & $70.36 \pm 3.44$ & $69.91 \pm 7.96$ & $\mathrm{NS}$ \\
\hline
\end{tabular}

All study and control children were subjected to a procedure of noninvasive recordings of electrocardiographic signals with the use of the body surface potential mapping (BSPM) method, giving a precise insight into the cardioelectric field. BSPM registrations were performed in the 87lead HPM-7100 system (Fukuda Denshi, Tokyo, Japan) [8]. The electrode standardized array was applied using one-use electrodes placed on 13 adhesive strips mounted in rows on the anterior and posterior thoracic surfaces (designated on the maps the letters A to M), as displayed in Fig. 1.

Using the raw electrocardiographic data obtained from the ECG signals recorded simultaneously from the 87 electrode sites, maps presenting ventricular activation time (VAT maps) were created. In the present study, VAT was determined by a computer program at each thoracic lead as the time taken from the onset of ventricular depolarization (i.e., onset of the QRS complex) to the peak of the R wave.



Fig. 1 Scheme of electrodes placement on the thoracic surfaces
In the next step, the difference map, which reflects the statistically significant differences between the two groupmean VAT maps (i.e., averaged for both control and patient group), was established. Differences were calculated through comparing the VAT values at each of the 87 electrode sites in the two group-mean isochrone maps.

The values of the difference map were obtained by calculating differences of the averaged values of ventricular activation time, taking into account the confidence interval at a significance level $\alpha=0.05$, at every recording site for both of the groups examined. The procedure was applied using EXCEL software. If the determined difference fell within the confidence interval, meaning that it did not differ significantly from zero, it was marked on the map as a null value. Other difference values, positive or negative, were significantly different from zero. Positive differences express a delay in the electrocardiographic signal reaching the measuring thoracic electrodes.

In addition to the quantity assessment mentioned above, a quality evaluation of the two group-mean VAT maps was performed by visual comparison of the distribution of the isochrone lines on the maps.

The parents of all children enrolled in the present study gave their informed consent to the procedures to be performed. The investigation protocol was approved by the local Bioethics Committee.

Table 1 displays data obtained from the comparisons performed between the different groups using the nonparametric Mann-Whitney $U$ test for the same distribution variables. A value of $p<0.05$ was considered to be statistically significant.

Probability values of VAT at each of the 87 measuring points were calculated using the Welch's test (generalized Student's $t$ test) and are presented in Table 2 . 
Table 2 Distribution of probability values

\begin{tabular}{llllllll}
\hline \multicolumn{7}{l}{ Number of electrodes } & \\
Strip electrodes & 1 & 2 & 3 & 4 & 5 & 6 & 7 \\
\cline { 2 - 8 } A & 0.001 & 0.001 & 0.001 & 0.001 & 0.001 & 0.001 & 0.001 \\
B & NS & NS & NS & 0.001 & 0.001 & 0.001 & 0.001 \\
C & NS & NS & NS & 0.001 & 0.001 & 0.001 & 0.001 \\
D & NS & NS & NS & NS & NS & 0.001 & 0.001 \\
E & NS & NS & NS & NS & NS & NS & 0.001 \\
F & NS & NS & NS & NS & NS & NS & 0.001 \\
G & NS & NS & NS & 0.001 & 0.001 & 0.001 & 0.001 \\
H & NS & NS & 0.001 & 0.001 & 0.001 & 0.001 & 0.001 \\
I & NS & 0.001 & 0.001 & 0.001 & 0.001 & 0.001 & 0.001 \\
J & 0.001 & 0.001 & 0.001 & 0.001 & 0.001 & 0.001 & 0.001 \\
K & 0.001 & 0.001 & 0.001 & 0.001 & 0.001 & 0.001 & 0.001 \\
L & 0.001 & 0.001 & 0.001 & 0.001 & 0.001 & 0.001 & 0.001 \\
M & 0.001 & 0.001 & 0.001 & 0.001 & 0.001 & 0.001 & 0.001 \\
A & 0.001 & 0.001 & 0.001 & 0.001 & 0.001 & 0.001 & 0.001 \\
\hline
\end{tabular}

\section{Results}

In healthy individuals, ventricular activation starts from the atrio-ventricular node, then proceeds via the His bundle branches, right and left, and finally reaches the myocardium through the Purkinje fibers (see Fig. 2).

On the VAT map averaged for the control children (Group $\mathrm{C}$ ), the shortest measurable activation time (from 1 to $17 \mathrm{~ms}$ ) is observed in the upper and middle portions of the anterior torso, along the mid-anterior line and adjacent lines (D, E, G) as well as in the upper left back (between the left posterior axillary line and the left scapular line).

The further isochrones of values up to $30 \mathrm{~ms}$ extend backward, spreading quite evenly toward the left and right parts of the thorax. Some of the isochrones on the left thorax comprise its upper section and slowly migrate to the back. Below that region, the range of isolines up to $40 \mathrm{~ms}$ occurs. Then, in the interval time from 40 to $50 \mathrm{~ms}$, isochrone lines cover not only the lower right anterior thorax, but also considerable areas of the thorax reaching the left posterior scapular line.

In the next phase of activation propagation, lasting up to $60 \mathrm{~ms}$, isochrones appear in the lower right anterior thorax, reaching the right midaxillary line, and in the back from the left posterior scapular line to, once again, the right midaxillary line. The final time interval, up to $80 \mathrm{~ms}$, finishing the graphical representation of ventricular activation trajectory, takes place over the upper left back and trespasses into the right upper anterior part of the thorax.

When analyzing the VAT map of healthy children, one can see that the area of the heart activated at first is a subendocardial layer of the left bottom surface of the intraventricular septum and a small portion of the free wall of the left ventricle. Then, the activation wave front wanders from the left to the right side of the intraventricular septum. In the next step, the activation extends over the right septal surface and subendocardial surfaces of the right and left ventricle free walls in the periapical region. Subsequently, free ventricular walls are activated, but within the left ventricle (because of its larger mass) this process lasts longer. In the final phase of ventricular depolarization, the wave front moves clearly upwards reaching the basal sections of the heart, which is seen on the VAT maps in the right portion of both the anterior and posterior surfaces of the thorax.

In 18 out of 22 patients with CKD (Group S) the distribution of isochrone lines in VAT maps differed from the control map (Fig. 3).

In these cases the onset of ventricular activation is observed in the area situated between the anterior midsternal line and both right and left parasternal lines. Isochrones appearing in the time interval up to $40 \mathrm{~ms}$ go toward the bottom of the anterior thorax. Starting from $50 \mathrm{~ms}$, on the left side isolines cross through the lower anterior thorax to the left axillary line, whereas on the right side, they reach to the right midclavicular line. Next, isochrones wander upward, to the middle part of the back, and on the right side come up to the right anterior axillary line. In this zone, there are some delays in activation propagation caused by disturbances in the left anterior fascicle of the His bundle. Up to $80 \mathrm{~ms}$, isochrones reach the upper back as well the right anterior portion of the thorax, which indicates the end of the ventricular depolarization process.

The distribution of VAT maps, assessed both visually and by comparing the VAT values at each of the measuring 
Fig. 2 Ventricular activation time (VAT)-mean map from the control children (C group). a On plane; 3-dimensional view a

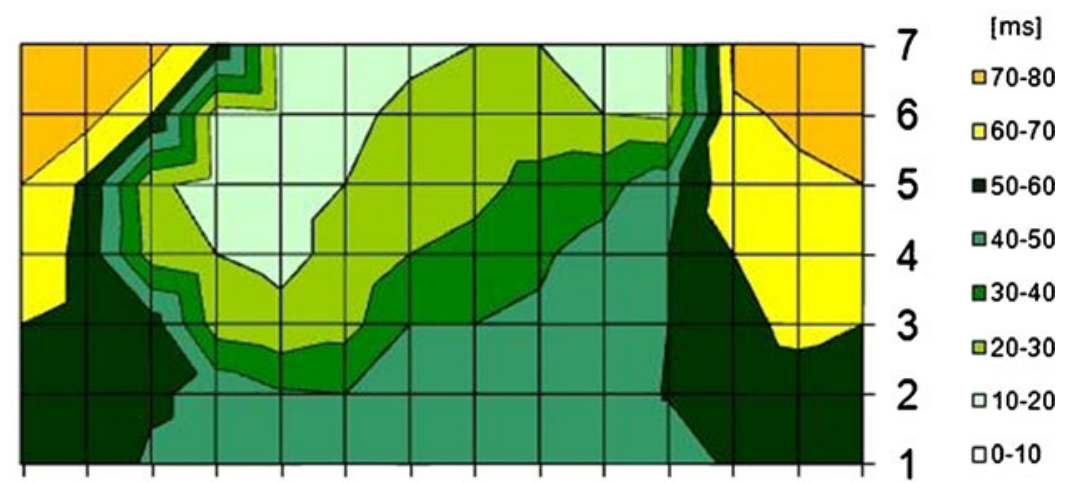

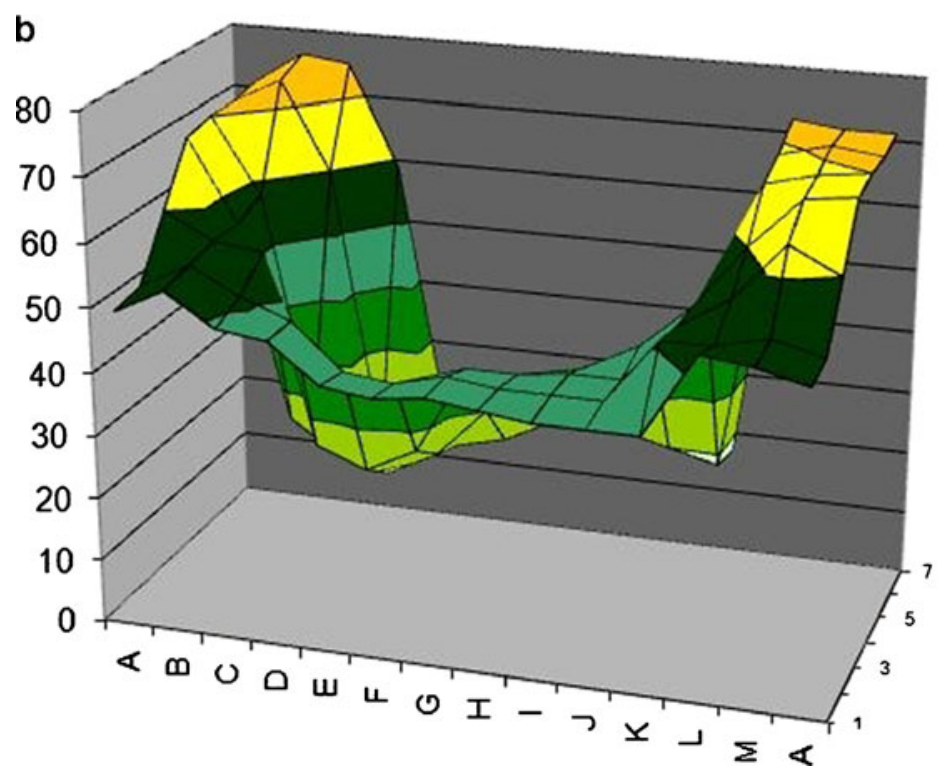

[ms]

ם70-80

व60-70

- $50-60$

$\square 40-50$

- $30-40$

$\square 20-30$

व10-20

व0-10

points, obtained from 18 child patients with CKD, is concordant with the pattern map previously established in our laboratory for cases with clinically diagnosed left anterior fascicular block of the His bundle (LAFB). As was seen on VAT maps taken from those CKD patients, the earliest ventricular depolarization, as in normal children, occurs in the subendocardial layer of the left lower intraventricular septum. Then, the activation wave front turns through the intraventricular septum from left to right. At that time, in the right parasternal region, short-term $\mathrm{R}$ waves appear. Up to $30 \mathrm{~ms}$, the activation state comprises the right surface of the intraventricular septum as well as the subendocardial layer of the free wall of the right ventricle. Because of LAFB, the free wall of the left ventricle is depolarized with a delay, using a collateral pathway conducting through the posterior fascicle of the bundle of His. In the final stage, the activation wave front reaches the anterior and lateral segments of the left ventricle, and then, still directing to the left, upward, and backward, comprises the remaining portions of the free wall of the left ventricle.

It is worth noting that the four remaining patients with CKD whose VAT maps showed physiological intraventricular conduction were at the second stage of CKD and presented relatively high eGFR values (range: $65.5 \pm$ $3.69 \mathrm{ml} / \mathrm{min} / 1.73 \mathrm{~m}^{2}$ ).

Application of the map of statistically significant differences makes it possible to compare distributions of the group-mean VAT maps taken from the two groups being examined, i.e., children with CKD treated conservatively and normal children. The difference map displays graphically the areas of discriminative divergence between the two group-mean VAT maps obtained from the two groups under consideration.

Analysis of the difference map revealed that both for the LAFB cases and for the control group, the first part of the ventricular activation trajectory up to the bundle division, as reflected on the VAT maps, is comparable; hence, there 
Fig. 3 VAT-mean map from the children with chronic kidney disease (CKD) treated conservatively ( $\mathrm{S}$ group) displaying a pattern of early left anterior fascicle block. a On plane; b 3-dimensional view a

[ $\mathrm{ms}$ ]
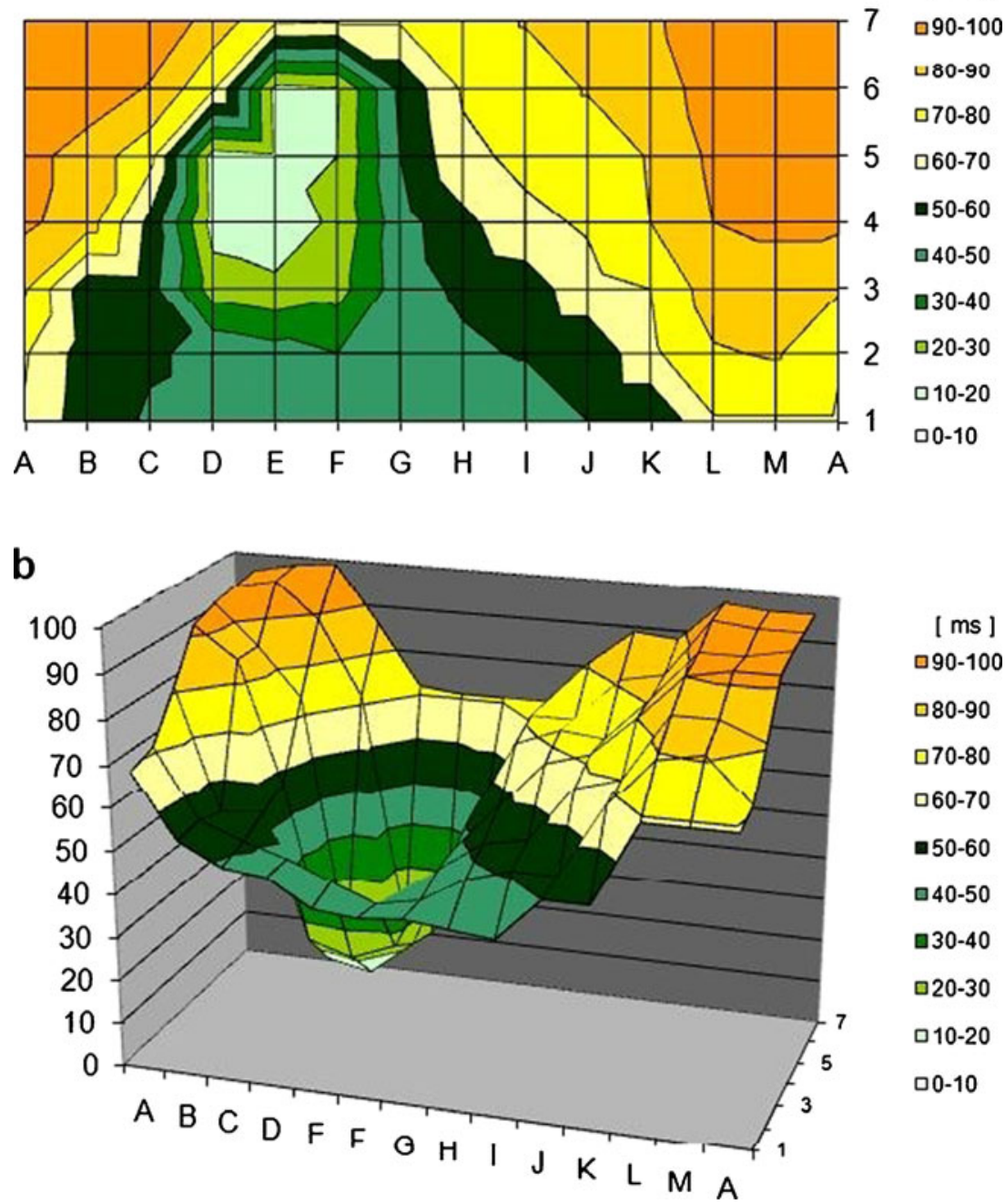

[ $\mathrm{ms}$ ]

व90-100

$\square 80-90$

$\square 70-80$

व60-70

- $50-60$

$\square 40-50$

$\square 30-40$

व20-30

व10-20

$\square 0-10$ was a lack of significant differences in an extensive region of the entire lower anterior, middle, and posterior thorax (Fig. 4).

In turn, ventricular activation time measured in the upper regions, from the right midclavicular line through the left axillary line, up to the right scapular line, is significantly prolonged in CKD cases, compared with the corresponding region in the control group. Isolines of values from $10 \mathrm{~ms}$ to $50 \mathrm{~ms}$ travel upward to the middle part of the back. The isoline with the highest value of $62 \mathrm{~ms}$ appears at the electrodes $\mathrm{J} 7$ and $\mathrm{K} 7$ located in the left portion of the thorax.

Probability values of VAT at each of the 87 measuring points are presented in Table 2 .

Taking into consideration the biochemical findings, in children with CKD, the serum iPTH concentration showed a statistically significant increase compared with the controls $(p<0.001)$. As for inorganic phosphate, its mean level was within the normal range for all the study children, although an insignificant tendency for the higher values in the CKD children was observed. Calcium ions also generally fell within the normal laboratory range, but in the CKD patients $\mathrm{Ca}^{+2}$ levels turned out to be significantly lower than in the control subjects $(p<0.05)$.

\section{Discussion}

Chronic kidney disease in children can lead to impairments in the cardiovascular system [9, 10]. One of the constant components of these changes is disturbances in depolarization propagation within the intraventricular conduction system. Metabolic abnormalities, including increased uremic toxemia, can be a reason, as well as some conduction alterations caused by various cardiac conditions, like left ventricle hypertrophy, cardiomyocytes degeneration, calcification of the myocardium, and changes in vascular architecture [11].

Kozlíková et al., using the 32-electrode BSPM system, established a pattern of isochrone maps for a population of 
Fig. 4 Difference map: map presenting the significant differences in the mean VAT values between the patient group and the control group. a On plane; b 3-dimensional view a

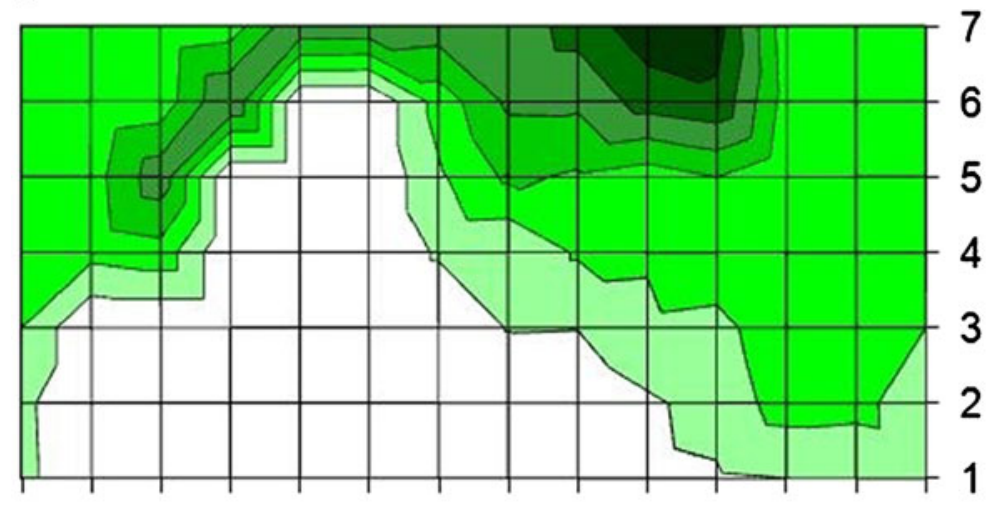



$\square 10-20$

$\square 0-10$



young, healthy subjects [12]. The isochrone maps, in accordance with our data from the 87-lead system, confirmed that under physiological conditions electrical impulses propagate to both ventricles through the atrioventricular node, the bundle of His, with its right and left branches, and finally via the Purkinje fibers to the myocardium.

Based on the distributions of the VAT maps obtained in our study, it was found that isochrones in children with CKD treated conservatively behaved in a different manner than in normal subjects. The conduction pathway seemed to be initially quite normal until it reached a region in the left anterior fascicle of the His bundle. In the further stages of activation propagation some delay of conduction was noted on the left side of the anterior thorax. This kind of conduction disturbance can lead to delayed depolarization of the left ventricle, which results in hemodynamic dyssynchrony and deteriorating systolic function of the heart $[13,14]$.

The above-described impairments yield a pattern of LAFB, which is a consequence of disturbances in this specific portion of the intraventricular conduction system. In such cases, the activation wave front is compelled to avoid the obstacle and proceeds at first through the posterior fascicle of the His bundle. This results in a slowing down of the depolarization process of both the anterior and lateral segments of the left ventricle, which are physiologically activated by the anterior fascicle [15]. Analyzing the isochrone map distributions, we observed changes suggesting LAFB in 18 out of 22 patients $(88 \%)$ with CKD who were treated conservatively. Only in the 4 remaining patients did the VAT maps present normal 
distributions of isochrones lines, confirming physiological intraventricular conduction.

Early and slight disturbances in the heart intraventricular conduction can be non-invasively detected only with a multielectrode recording system, like BSPM. BSPM has been shown to be the only non-invasive method of assessing the cardioelectric field from signals collected on the body surface, which is of higher sensitivity and specificity compared with the standard 12-lead ECG examination [16].

It is assumed that in patients with CKD numerous cardiovascular disorders are induced mainly by arterial hypertension and secondary hyperparathyroidism [9]. In fact, a significant increase in serum $\mathrm{PTTH}$ was found in our study group with $\mathrm{CKD}$, compared with the control group (91.00 \pm 5.21 vs $41.53 \pm 4.33 ; p<0.001$ ).

Considerable long-standing hypersecretion of intact parathyroid hormone could account for changes occurring in the intraventricular conduction system in CKD patients. iPTH is commonly considered one of the most serious uremic toxins. Elevated iPTH level may be a cause of calcification of coronary arteries. Relationships between serum iPTH levels and arteriosclerotic changes both in vivo and in vitro have been demonstrated $[17,18]$. Furthermore, increased iPTH concentration results in enhanced influx of $\mathrm{Ca}^{2+}$ ions into the cell cytoplasm and subsequent damage to mitochondria, which can result in dysfunction of the heart conduction system [19].

On the other hand, significantly lower total $\mathrm{Ca}^{+2}$ serum concentrations were found in our study patients $(9.65 \pm 0.71$ vs $10.02 \pm 0.34 ; p<0.05)$. It is known that hypocalcamia can lead to improper muscle contraction and distorted transmission of electrical impulses in cardiomyocytes [18, 19]. Calcium depletion compromises membrane calcium channel activity and inward flow of calcium ions to cardiomyocytes, which is prerequisite for the upstroke of the action potential and proper depolarization progress in the heart [20]. According to some investigators (e.g., Mitsnefes et al.), increased serum phosphate concentrations can also cause pathological alterations in the cardiovascular system [21]. However, in our study such contributions cannot be considered a pathomechanism of intraventricular conduction abnormalities, since no significant differences in phosphate levels were noted between the CKD patients and the control group.

A novel approach presented in our study was the use of a difference map showing significant differences between the VAT maps averaged for the groups of children examined: healthy subjects and CKD patients. The difference map, in a simple and quick visual manner, enables the assessment of the areas in which VAT values are delayed in relation to those established for the controls. In addition, this statistical technique facilitates recognition of precise sites of activa- tion propagation blockage within the heart's intraventricular conduction system.

Progression in the clinical course of CKD inevitably leads to serious heart conduction impairments as well as left ventricle hypertrophy and heart failure [22]. The difference map turned out to enhance the areas of intergroup VAT changes, which are of discriminative value in detecting intraventricular conduction disturbances. Therefore, the study children with CKD, in whom intraventricular conduction disturbances were diagnosed with BSPM, could be referred for early cardiac supervision.

Summing up, the results of our study suggest that BSPM, with its high sensitivity, enables early detection of intraventricular conduction abnormalities, which are not seen on the routine ECG. This allows us to perform a stratification of CKD patients and provide them with adequate management, in order to protect them from severe cardiac complications.

\section{Conclusions}

1. Intraventricular conduction system impairments within the left bundle branch may occur in children with moderate CKD.

2. BSPM enables the detection of heart conduction disturbances in children with CKD earlier than 12lead ECG.

3. The difference map derived from the isochrone groupmean maps precisely localizes the sites of disturbed conduction in the heart's intraventricular system.

Disclosure None of the authors declared any competing interests.

Open Access This article is distributed under the terms of the Creative Commons Attribution Noncommercial License which permits any noncommercial use, distribution, and reproduction in any medium, provided the original author(s) and source are credited.

\section{References}

1. Wu-Wong JR (2009) Potential for vitamin D receptor agonists in the treatment of cardiovascular disease. Br J Pharmacol 158:395412

2. Polak-Jonkisz D, Laszki-Szcząchor K, Purzyc L, Zwolińska D, Musiał K, Pilecki W, Rusiecki L, Janocha A, Kałka D, Sobieszczańska M (2009) Usefulness of body surface potential mapping for early identification of the intraventricular conduction disorders in young patients with chronic kidney disease. J Electrocardiol 42:165-171

3. Miyashita T, Okano Y (1995) Isochrone map, its implication and clinical usefulness. Nippon Rinsho 1:48-55 
4. Green LS, Abildskov JA (1995) Clinical application of body surface potential mapping. Clin Cardiol 18:24

5. Katayama K, Kamakura S, Shimomura K (1995) Relationship between prognosis and electrocardiographic characteristic in patients with left bundle branch block. Nippon Rinsho 1:132138

6. National Kidney Foundation (2008) K/DOQI clinical practice guidelines for chronic kidney disease: evaluation, classification, and stratification. Am J Kidney Dis 39:S1-S266

7. Schwartz GJ, Brion LP, Spitzer A (1987) The use of plasma creatinine concentration for estimating glomerular filtration rate in infants, children and adolescents. Pediatr Clin North Am 34:488493

8. Fukuda Denshi Co. Ltd (1990) Heart potential mapping system. Fukuda Denshi Co. LTD Bulletin, Tokyo

9. Chavers BM, Li S, Collins AJ, Herzog CA (2002) Cardiovascular disease in pediatric chronic dialysis patients. Kidney Int 62:648-653

10. Scharer K, Schmidt KG, Soergel M (1999) Cardiac function and structure in patients with chronic renal failure. Pediatr Nephrol 13:951-965

11. Robinson RF, Nahata MC, Sparks E, Daniels C, Batisky D, Hayes JR, Mahan J (2005) Abnormal left ventricular mass and aortic distensibility in pediartic dialysis patients. Pediatr Nephrol 20:64-68

12. Kozlíková K, Hulín I, Kneppo P, Sapáková E, Bakosová M, Molnárová Z (1993) Body surface isochrone maps of peak $R$ in normal adolescent girls. Physiol Res 42:99-102

13. Pastore CA, Tobias NM, Kaiser E, Samesima N, Martinelli FM, Pedrosa A, Nishioka S, Siqueira S, Lourenco UR, Atanes LM, Ramires JAF (2002) Electrocardiographic, vectocardiographic and body surface potential mapping analyses of patients with congestive heart failure and left bundle branch block, treated with biventricular pacing. Int J Bioelectromagnetism 4:115-116
14. Potse M, Linnenbank AC, Grimbergen CA (2002) Software design for analysis of multichannel intracardial and body surface electrocardiograms. Comput Meth Programs Biomed 69:225-236

15. Fuster Siebert M, Castillo Sánchez J, Domińguez Sánchez R (1980) Ventricular activation time at aVL and V6 leads in left anterior hemiblock. Rev Esp Cardiol 33:573-579

16. Kornreich F, MacLeod RS, Lux RL (2008) Supplemented standard 12-lead electrocardiogram for optimal diagnosis and reconstruction of significant body surface map patterns. J Electrocardiol 41:251256

17. Nakayama T, Ohtsuru A, Enomoto H, Namba H, Ozeki S, Shibata Y, Yokota T, Nobuyoshi M, Ito M, Sekine I, Yamashita S (1994) Coronary atherosclerotic smooth muscle cells overexpress human parathyroid hormone-related peptides. Biochem Biophys Res Commun 200:1028-1035

18. Jono S, Nishizawa Y, Shioi A, Morii H (1997) Parathyroid hormonerelated peptide as a regulator of vascular calcification. Its inhibitory action on in vitro calcification by bovine vascular smooth muscle cells. Arterioscler Thromb Vasc Biol 17:11351142

19. Rodriguez M, Lorenzo V (2009) Parathyroid hormone, a uremic toxin. Semin Dial 22:363-368

20. Lipscombe D (2002) L-type calcium channels. High and new lows. Circ Res 90:933-935

21. Mitsnefes MM, Kimball TR, Kartal J, Witt SA, Glascock BJ, Khoury PR, Daniels SR (2005) Cardiac and vascular adaptation in pediatric patients with chronic kidney disease: role of calciumphosphorus metabolism. J Am Soc Nephrol 16:2796-2803

22. Mehta A, Jain AC, Mehta MC, Billie M (2000) Usefulness of left atrial abnormality for predicting left ventricular hypertrophy in the presence of left bundle branch block. Am J Cardiol 85:354-359 